\title{
From Other Journals: A Review of Recent Articles in Pediatric Cardiology
}

\author{
Tarek Alsaied $^{1,2}$ (1) $\cdot$ Awais Ashfaq ${ }^{1,3}$
}

Received: 28 July 2020 / Accepted: 19 August 2020 / Published online: 3 September 2020

(c) Springer Science+Business Media, LLC, part of Springer Nature 2020

\begin{abstract}
In this review, we provide a brief description of recently published articles addressing topics relevant to pediatric cardiologists. Our hope is to provide a summary of the latest articles published recently in other journals in our field. The articles address (1) pulmonary valve repair in patients with tetralogy of Fallot and in patients with congenital pulmonary stenosis which seems to be a promising technique in selected patients, (2) the use of Losartan in patients with Marfan syndrome mostly in addition to beta blockers may result in a lower aortic root dilation rate and better clinical outcomes, (3) a summary of the characteristics of adults with congenital heart disease in the USA showed a wide variation in prevalence, associated morbidities, health care utilization and insurance type in different locations in the USA, (4) a large single center study confirmed that right ventricular morphology is associated with worse outcomes after the Fontan operation and that atrioventricular valve regurgitation is an important predictor of worse outcomes and precedes ventricular dysfunction post Fontan operation, (5) a large study describing the multi system inflammatory syndrome in children temporarily related to the COVID-19 pandemic in the USA showing that ventricular dysfunction is the most common cardiac manifestation (6) the reality of "limping to transplantation" showing that patients with one or more modifiable risk factors including mechanical ventilation, kidney or liver dysfunction are at significant increase risk post cardiac transplantation.
\end{abstract}

Keywords Pulmonary valve repair $\cdot$ Tetralogy of Fallot $\cdot$ Marfan syndrome $\cdot$ Adults with congenital heart disease $\cdot$ Heart transplantation $\cdot$ Multisystem inflammatory syndrome in children

In this review, we provide a brief description of recently published articles addressing topics relevant to pediatric cardiologists. Our hope is to provide a summary of the latest articles published in other journals in our field.

Tarek Alsaied

tarek.alsaied@cchmc.org

1 Heart Institute, Cincinnati Children's Hospital Medical Center, 3333 Burnet Avenue, Cincinnati, OH 45229, USA

2 Department of Pediatrics, University of Cincinnati College of Medicine, Cincinnati, OH, USA

3 Heart Institute, Johns Hopkins All Children's Hospital, St. Petersburg, FL, USA

\section{Secondary Repair of Incompetent Pulmonary Valves After Previous Surgery or Intervention: Patient Selection and Outcomes [1]}

Pulmonary valve (PV) regurgitation (PR) is common after intervention for tetralogy of Fallot and congenital pulmonary stenosis. PV repair is an alternative to replacement (PVR) although selection criteria are not established. This study sought to clarify preoperative variables associated with successful PV repair and to compare outcomes between repair and PVR. This was a single center retrospective study that assessed patients who underwent surgery for PR from 2010 to 2017 by a single surgeon. The PV annulus and leaflets were measured on the preoperative echocardiogram and magnetic resonance imaging (CMR), and the primary predictor variable was leaflet area indexed to ideal PV annulus area (iPLA) by magnetic resonance imaging. PV repair and PVR groups were compared using multivariable logistic regression, and with a conditional inference tree. The study 
included 85 patients, 31 (36\%) underwent PV repair. By multivariable analysis, longer PV total leaflet length $\left(\mathrm{cm} / \mathrm{m}^{2}\right)$ $(\beta=3.00$, standard error $[\mathrm{SE}]=0.82, P<0.001)$, larger $\mathrm{PV}$ annulus $z$ score $(\beta=1.34, \mathrm{SE}=0.39, P=0.001)$, and larger iPLA $(\beta=8.13, \mathrm{SE}=2.62, P=0.002)$ were associated with repair. iPLA of 0.90 or greater was $91 \%$ sensitive and $83 \%$ specific for achieving PV repair. At a median of 4.1 years follow-up, there was greater freedom from significant $P R$ in the PV repair group (log rank $P=0.008$ ). Patients with an iPLA $>0.9$, and those with an iPLA between 0.7 and 0.9 with a PV annulus $z$ score $>0$ should be considered for a native PV repair. At midterm follow-up, patients with a $\mathrm{PV}$ repair were not more likely to develop PR or to require reintervention when compared with patients undergoing PVR. This is a promising new technique in selected patients (Table 1).

\section{Long-Term Clinical Outcomes of Losartan in Patients with Marfan Syndrome: A Follow Up of the Multicenter COMPARE Trial [2]}

The Marfan syndrome is a connective tissue disorder caused by a mutation in the fibrillin 1 gene which results in structural dysfunction in the aortic media layer due to upregulation in tissue growth factor beta (TGF-b). This results in aortic dilation/aneurysm formation and increases the risk for aortic dissection. A previous randomized controlled trial, the COMPARE trial, demonstrated a small but significant effect of 3 years treatment with losartan on the rate of aortic root enlargement as compared to placebo in Marfan syndrome [3]. The aim of the current study was to investigate the long-term clinical outcomes after losartan treatment.

Table 1 Summary of the six studies in this review

\begin{tabular}{|c|c|}
\hline Author & Study summary \\
\hline Adamson et al. & $\begin{array}{l}\text { Pulmonary valve repair in tetralogy of Fallot and congenital pulmonary stenosis } \\
85 \text { patients, } 31 \text { underwent valve repair and the rest valve replacement } \\
\text { Patients with longer valve leaflets, higher annular } z \text {-score and larger valve area were better candidates for repair } \\
\text { At a median follow up of } 4.1 \text { there was greater freedom from regurgitation in the repair group } \\
\text { Pulmonary valve repair is a good option in selected candidates with non-inferior results compared to replacement }\end{array}$ \\
\hline Van Andel et al. & $\begin{array}{l}\text { Long-term outcomes of losartan in adult patients with Marfan syndrome } \\
75 \text { patients on losartan for } 8 \text { years and } 78 \text { controls. Most patients were on beta blockers } \\
\text { Losartan group is younger } 34 \text { vs } 41 \text { years } \\
\text { Losartan group with less death and dissection after adjusting for age and beta-blocker use } \\
\text { Losartan can decrease the incidence of clinical complication in combination with beta blockers in Margan syndrome }\end{array}$ \\
\hline Gurvitz et al. & $\begin{array}{l}\text { Characteristics of adults with congenital heart disease (CHD) in the USA } \\
\text { Demographics, health care use and insurance status was studied for adults with CHD } \\
3 \text { locations: Emory University, Massachusetts and New York State Health Department } \\
\text { Wide variation in Medicade use, number of encounters and prevalence of comorbidities } \\
\text { High rates of cardiac and non-cardiac comorbidities } \\
\text { In the absence of an integrated health care system, studying trends between states remains challenging. This study shows wide } \\
\text { variability which can be due to true variability or difference in coding and data collection methods }\end{array}$ \\
\hline Moon et al. & $\begin{array}{l}\text { Ventricular morphology and outcomes after the Fontan procedure } \\
1162 \text { patients who underwent the Fontan procedure at single institution } \\
\text { Transplant and takedown-free survival of } 91 \%, 75 \% \text {, and } 71 \% \text { at } 10,20 \text {, and } 25 \text { years, respectively } \\
\text { Atrioventricular valve regurgitation preceded ventricular dysfunction and with right ventricular morphology were independent } \\
\text { risk factors (hazard ratio } 4.3 \text { and } 2.4 \text {, respectively) } \\
\text { Atrioventricular valve regurgitation precede ventricular dysfunction and in addition to right ventricular morphology are risk } \\
\text { factors for worse outcomes post Fontan }\end{array}$ \\
\hline Feldstein et al. & $\begin{array}{l}\text { The multisystem inflammatory syndrome in children (MIS-C) in the USA } \\
186 \text { patients from } 53 \text { centers and } 26 \text { States. Median age } 8.3 \text { years } \\
\text { Gastrointestinal involvement was most common }(92 \%) \text {, followed by cardiovascular ( } 80 \%) \\
\text { Ventricular dysfunction is the most common cardiac manifestation } 38 \% \text { and coronary aneurysms } 8 \% \\
\text { Hemodynamic support and immunomodulatory therapies are the primary treatments. Most children recover from MIS-C, but } \\
\text { medium- and long-term sequelae are unknown }\end{array}$ \\
\hline Riggs et al. & $\begin{array}{l}\text { The reality of limping to pediatric heart transplantation } \\
4101 \text { transplants from UNOS, } 1459 \text { patients }(36 \%) \text { had } 1 \text { or more modified risk factors (MRFs) } \\
\text { There was a decrease in 1-year survival with additional MRFs up to a } 9.1 \text { times increased risk of death in an infant with con- } \\
\text { genital heart disease (CHD) } \\
\text { Patients "limping to transplant" with multiple risk factors demonstrates decreasing early survival relative to those without } \\
\text { other end-organ dysfunction }\end{array}$ \\
\hline
\end{tabular}

CHD congenital heart disease 
The COMPARE trial included patients from four Dutch academic hospitals who were above the age 18 years in 2008 and 2009. Patients were excluded if they have a previous dissection, more than one vascular prosthesis or had an aortic root $>50 \mathrm{~mm}$ at inclusion. The losartan dose started at $50 \mathrm{mg}$ and increased to $100 \mathrm{mg}$ daily which was achieved in $54 \%$ of the patients. Of the original 233 patients included in the COMPARE trial 220 could be traced and analyzed after a median follow up of 8 years. Of note $27 \%$ of the patients had a previous aortic root replacement before the COMPARE trial. The baseline aortic root dimension at enrollment was $44 \mathrm{~mm}$ by cardiac MRI. Clinical endpoints, defined as all-cause mortality, aortic dissection/rupture, elective aortic root replacement, reoperation, and vascular graft implantation beyond the aortic root and a composite end point of all the above, were compared between the two groups.

The original COMPARE trial showed in the losartan group a slightly lower systolic blood pressure by $3 \mathrm{mmHg}$ and a small but significant decrease in aortic root dilation $(0.77 \mathrm{~mm}$ versus $1.35 \mathrm{~mm})$ which remained significant in the subsets with history of aortic root replacement.

After the COMPARE trial period of 3 years, study subjects chose to continue their losartan medication or not. 75 patients continued losartan medication, whereas 78 patients, originally allocated to the control group, never used losartan after inclusion. There was no difference in baseline characteristics of the two groups except for age at inclusion (losartan 34 years, control 41 years; $P=0.031$ ), and $\beta$-blocker use (losartan $81 \%$, control $64 \% ; P=0.022$ ). A pathological FBN1 mutation was present in $76 \%$ without a difference between the groups. Five deaths, 14 dissections, 23 root replacements, 3 reoperations, and 3 vascular graft implantations beyond the aortic root occurred during follow-up. Except for aortic root replacement, all endpoints occurred in patients with an operated aortic root. Patients who used losartan during the entire followup period showed a reduced number of events compared to the control group (death: 0 vs. $5, P=0.014$; aortic dissection: 3 vs. $11, P=0.013$; elective aortic root replacement: 10 vs. $13, P=0.264$; reoperation: 1 vs. $2, P=0.463$; vascular graft implantations beyond the aortic root 0 vs. 3 , $P=0.071$; and composite endpoint: 14 vs. $26, P=0.019$ ). These results remained similar when corrected for age and beta-blocker use in a multivariate analysis. The study concluded that combined losartan with beta-blocker have a clinical benefit in Marfan syndrome.

To summarize this follow-up study of the COMPARE trial shows that the use of losartan mostly in combination with beta-blocker resulted in slower rate of aortic root dilation and better clinical outcomes. Of note is that most of these patients taking losartan were younger although age was adjusted for on multivariable analysis.

\section{Characteristics of Adults with Congenital Heart Disease in the USA [4]}

Adults with congenital heart disease (CHD) are a growing and aging population with high incidences of cardiac and non-cardiac comorbidities and high rates of health care utilization. This study investigated demographics, comorbidities and heath care use of adults above the age of 20 years and below 64 years with CHD in the USA. The study included patients at three locations in the USA and recorded data between 2008 and 2010 using ICD-9 codes. These locations were Emory University including five counties around Atlanta, GA, the Massachusetts and the New York State Department of Health. Patients with isolated atrial level communication were excluded. The study looked at demographics, insurance type, comorbidities and all encounter data were collected while the Census data were used as a denominator. Up to $20 \%$ of adults with CHD had severe CHD and more than $50 \%$ had a comorbidity with variation between the locations. There was also significant variability in the type of insurance and the proportion of encounters in patients with CHD across the three locations. Massachusetts had a large proportion of Medicaid (75\%) and Emory University and New York had large proportions of private insurance (44\% and 67\%, respectively). There was a wide variability in estimated proportions of adults with CHD-coded health care encounters by location, with 1.2 (Emory University), 10 (Massachusetts), and 0.6 (New York) per 1000 adults. Due to the lack of a national registry for adults with CHD and the lack of an integrated health care system, understanding regional trends represent a significant challenge for health care professionals, epidemiologists and health policy makers. This study is a big step forward and highlights significant regional variations. Due to the nature of the data these variations could represent true variations but could also represent differences in coding and data collection. The study is an important step to understand the burden of CHD among adults and highlights the importance of a national registry for this patient population.

\section{Ventricular Morphology and Outcomes After the Fontan Procedure [5]}

There is a rapid increase in the number of patients with a Fontan circulation and Fontan circulatory failure remains a significant concern in this population [6]. This study hypothesized that dysfunction of the single right ventricle (RV) and right atrioventricular valve regurgitation 
(AVVR) increases over time and adversely impacts late outcomes following a Fontan operation. This large retrospective single center study evaluated the impact of ventricular morphology and atrioventricular valve function on late outcomes following the Fontan procedure. The study included all patients undergoing the Fontan procedure between 1985 and 2018 at University of Michigan. Data regarding death or heart transplantation were obtained from the National Death Index and the Scientific Registry of Transplant Patients. The study included a total of 1162 patients who underwent the Fontan procedure during the study period, with transplant and takedown-free survival of $91 \%, 75 \%$, and $71 \%$ at 10,20 , and 25 years, respectively. As expected, morphologic RV was an independent risk factor for transplant and takedown-free survival (hazard ratio $2.4 ; P=0.008$ ). Interestingly, AVVR preceded ventricular dysfunction in most cases and was associated with the development of ventricular dysfunction after Fontan (odds ratio 4.3; 95\% confidence interval 2.7-6.7; $P<0.001)$. AVVR and ventricular dysfunction appeared progressive after Fontan, particularly patients with RV morphology (AVVR: $P<0.0001$, ventricular dysfunction: $P<0.0001$ ). The study concluded that morphologic RV is negatively associated with long-term survival following the Fontan operation. There is a tendency for progressive AVVR and ventricular dysfunction, particularly in patients with systemic RVs. Although it is intuitive that systemic $\mathrm{RV}$ will result in worse outcomes in patients with single ventricle, there is still a debate about that and the data are inconsistent. This study adds to the body of literature suggesting worse outcomes in patients with systemic RV. The study shows that similar to patients with congenitally corrected transposition where tricuspid regurgitation is a major contributor to morbidity and ventricular dysfunction, AVVR preceded ventricular dysfunction and worsen the outcomes in patients with a Fontan circulation. The study also shows similar long-term survival to other populations with a Fontan circulation with a 25 years survival of $\sim 70 \%$ and a progressive risk of AVVR and ventricular dysfunction post Fontan [7, 8]. While the Kaplan Meir curve in this study for ventricular morphology is different from previous studies as this study shows similar curves for the first 10 years and the two curves separate after 10 years while in previous studies mortality for patients with RV seems to be an early event then the curves become parallel [9]. Figure 3 in this paper shows nicely the temporal relationship between AVVR and ventricular dysfunction and clarifies that AVVR typically precedes ventricular dysfunction. Finally similar to previous studies tricuspid and common atrioventricular valves are more likely to develop AVVR compared to single mitral valve.

\section{The Multisystem Inflammatory Syndrome in Children (MIS-C) in the USA [10]}

To date, Coronavirus disease-19 (COVID-19) has infected more than 9 million people and resulted in over 450,000 deaths [11]. Initial reports indicated that children were largely spared from the most severe symptoms in COVID19 and were often asymptomatic [12]. However, in late April 2020, reports emerged from the United Kingdom, of children presenting with fever and hyperinflammation temporally associated with COVID-19 and with clinical features similar to incomplete Kawasaki disease (KD), toxic shock syndrome, macrophage activating syndrome, cytokine release syndrome or bacterial sepsis [13]. This new syndrome has been named Multisystem Inflammatory Syndrome in Children (MIS-C) by the Centers for Disease Control (CDC). This study investigated the epidemiology and clinical course of MIS-C and its temporal association with COVID-19. The study was a multi-institutional study including 53 centers in the USA from March 15 to May 20, 2020. The case definition was patients $<21$ years hospitalized with a febrile illness $>24 \mathrm{~h}$ with a laboratory evidence of inflammation and multi-organ involvement in the setting of an infection with the severe acute respiratory syndrome coronavirus 2 (SARS-COV-2) either by PCR or antibody testing or exposure to patients with COVID-19. The study included a total of 186 patients with MIS-C in 26 states. The median age was 8.3 years and $62 \%$ were male. Most patients (70\%) were positive for SARS-CoV-2 based on RT-PCR or antibody testing, with the remainder having exposure to patients with COVID-19. Consistent with previous smaller studies, gastrointestinal organ system involvement was most common (92\%), followed by cardiovascular ( $80 \%)$, hematologic (76\%), mucocutaneous (74\%), and respiratory $(70 \%)$. The median duration of hospitalization was 7 days, while $80 \%$ of patients required intensive care, $20 \%$ received mechanical ventilation, $48 \%$ received vasoactive support, and $2 \%$ died. Ventricular dysfunction was commonly seen with a ventricular ejection fraction of $30-55 \%$ in $33 \%$ of patients and $<30 \%$ in $5 \%$ of patients. Coronary artery aneurysms $(z$-scores $\geq 2.5)$ were seen in $8 \%$ of patients. Intravenous immune globulin was used in $77 \%$ of patients, glucocorticoids in $49 \%$, and interleukin- 6 or 1RA inhibitors in $20 \%$. The authors conclude that MIS-C in children associated with SARS-CoV-2 led to serious and life-threatening illness in previously healthy children and adolescents. Similar to previous smaller studies, left ventricular dysfunction is the most common cardiac manifestation of MIS-C, followed by coronary artery aneurysm. Hemodynamic support and immunomodulatory therapies are the primary treatments. Most children recover from MIS-C, but medium- and longterm sequelae are unknown. 


\section{The Reality of Limping to Pediatric Heart Transplantation [14]}

Improvements in surgical technique, critical care, and early repair for congenital heart disease (CHD) have led to improved outcomes with heart transplantation, often used as a salvage procedure after failed palliation, especially in infants. This study questioned if we sometimes wait too long as some of these patients accumulate morbidities and aimed to identify the reality of survival after heart transplantation in patients "limping to transplant" with common risk factors. The study used the UNOS data set from 2000 to 2017 and looked at recipients $<18$ years. Modifiable risk factors (MRFs) of mechanical ventilation, renal dysfunction, and liver dysfunction at transplant and nonmodifiable risk factors of infancy at listing or CHD were examined. One-year posttransplant survival was analyzed with logistic regression. The study found that of 4101 transplants, 1459 patients (36\%) had 1 or more MRFs. There was a decrease in 1-year survival with additional MRFs up to a 9.1 times increased risk of death in an infant with CHD. An example will be a noninfant without CHD and no MRFs had a 95\% 1-year survival, in contrast to an intubated patient with CHD without other end-organ dysfunction, who had 1-year survival of $76 \%$, which decreased to $58 \%$ if they were an infant and also had renal dysfunction. The authors concluded that patients "limping to transplant" with multiple risk factors demonstrates decreasing early survival relative to those without other end-organ dysfunction. It is imperative that we have transparent discussions about expected outcomes with these families and identify ways to optimize patients' conditions through other supportive avenues to improve posttransplant outcomes. In addition mechanical support with ventricular assist devices may help improve MRFs before transplantation. Providers need be aware of realistic expectations for certain groups of pediatric heart transplant candidates to facilitate transparent conversations with families and to stimulate the search for better methods of optimizing patient condition before transplantation. This data will also be helpful when adjusting for these risk factors in pediatric transplant registries.

Author Contributions Tarek Alsaied and Awais Ashfaq reviewed the literature and wrote the review.

Funding Not applicable.

Data Availability Not applicable.

Code Availability Not applicable.

\section{Compliance with Ethical Standards}

Conflict of interest Not applicable.

Ethical Approval Not applicable.

Consent to Participate Not applicable.

Consent for Publication Not applicable.

\section{References}

1. Adamson GT, McElhinney DB, Lui G, Meadows AK, Rigdon J, Hanley FL, Maskatia SA (2020) Secondary repair of incompetent pulmonary valves after previous surgery or intervention: patient selection and outcomes. J Thorac Cardiovasc Surg 159(6):2383. e2-2392.e2

2. van Andel MM, Indrakusuma R, Jalalzadeh H, Balm R, Timmermans J, Scholte AJ, van den Berg MP, Zwinderman AH, Mulder BJM, de Waard V et al (2020) Long-term clinical outcomes of losartan in patients with Marfan syndrome: follow-up of the multicentre randomized controlled COMPARE trial. Eur Heart J. https ://doi.org/10.1093/eurheartj/ehaa377

3. Groenink M, den Hartog AW, Franken R, Radonic T, de Waard V, Timmermans J, Scholte AJ, van den Berg MP, Spijkerboer AM, Marquering HA et al (2013) Losartan reduces aortic dilatation rate in adults with Marfan syndrome: a randomized controlled trial. Eur Heart J 34(45):3491-3500

4. Gurvitz M, Dunn JE, Bhatt A, Book WM, Glidewell J, Hogue C, Lin AE, Lui G, McGarry C, Raskind-Hood C et al (2020) Characteristics of adults with congenital heart defects in the United States. J Am Coll Cardiol 76(2):175-182

5. Moon J, Shen L, Likosky DS, Sood V, Hobbs RD, Sassalos P, Romano JC, Ohye RG, Bove EL, Si MS (2020) Relationship of ventricular morphology and atrioventricular valve function to long-term outcomes following Fontan procedures. J Am Coll Cardiol 76(4):419-431

6. Alsaied T, Bokma JP, Engel ME, Kuijpers JM, Hanke SP, Zuhlke L, Zhang B, Veldtman GR (2017) Predicting long-term mortality after Fontan procedures: a risk score based on 6707 patients from 28 studies. Congenit Heart Dis 12(4):393-398

7. King G, Ayer J, Celermajer D, Zentner D, Justo R, Disney P, Zannino D, d'Udekem Y (2019) Atrioventricular valve failure in Fontan palliation. J Am Coll Cardiol 73(7):810-822

8. Pundi KN, Johnson JN, Dearani JA, Pundi KN, Li Z, Hinck CA, Dahl SH, Cannon BC, O'Leary PW, Driscoll DJ et al (2015) 40-year follow-up after the Fontan operation: long-term outcomes of 1,052 patients. J Am Coll Cardiol 66(15):1700-1710

9. Dennis M, Zannino D, du Plessis K, Bullock A, Disney PJS, Radford DJ, Hornung T, Grigg L, Cordina R, d'Udekem Y et al (2018) Clinical outcomes in adolescents and adults after the Fontan procedure. J Am Coll Cardiol 71(9):1009-1017

10. Feldstein LR, Rose EB, Horwitz SM, Collins JP, Newhams MM, Son MBF, Newburger JW, Kleinman LC, Heidemann SM, Martin AA et al (2020) Multisystem inflammatory syndrome in US children and adolescents. N Engl J Med 383(4):334-346

11. Sohrabi C, Alsafi Z, O'Neill N, Khan M, Kerwan A, Al-Jabir A, Iosifidis C, Agha R (2020) World Health Organization declares global emergency: a review of the 2019 novel coronavirus (COVID-19). Int J Surg 76:71-76

12. Cases in U.S. Centers For Disease Control and Prevention. https ://www.cdcgov/coronavirus/2019-ncov/cases-updates/cases-inushtml 
13. Riphagen S, Gomez X, Gonzalez-Martinez C, Wilkinson N, Theocharis P (2020) Hyperinflammatory shock in children during COVID-19 pandemic. Lancet 395(10237):1607-1608

14. Riggs KW, Zafar F, Lorts A, Chin C, Bryant R 3rd, Tweddell JS, Morales DLS (2020) The reality of limping to pediatric heart transplantation. J Thorac Cardiovasc Surg 159(6):2418.e24112425.e2411
Publisher's Note Springer Nature remains neutral with regard to jurisdictional claims in published maps and institutional affiliations. 